DOI: 10.12731/2227-930X-2018-4-122-136

УДК 656.212.5

\title{
О ПОДХОДЕ К ОПРЕДЕЛЕНИЮ \\ НЕКОТОРЫХ КИНЕМАТИЧЕСКИХ ПАРАМЕТРОВ ДВИЖЕНИЯ ВАГОНА НА ТОРМОЗНЫХ ПОЗИЦИЯХ СОРТИРОВОЧНЫХ ГОРОК
}

\section{Туранов Х.Т., Гордиенко А.А., Саидивалиев Ш.У.}

При проектировании сортировочных горок, выборе ее оптимальных параметров большое значение имеют точные величины кинематических параметров движения вагонов, отиепов на всех ее участках. Проведенный анализ существующей методики расчета сортировочных горок показывает, что используемые формуль содержат значительное количество некорректностей и не содержит некоторых выражений для определения важных кинематических величин. В статье приведено математическое описание движения вагона на участках тормозных позиций сортировочной горки с использованием теоремы об изменении кинетической энергии для несвободной материальной точки в конечной форме. Данный подход позволяет решить ранее трудноразрешимую задачу по выводу формулы пути торможения вагона на участках тормозных позиций сортировочных горок. Полученная математическая модель апробирована на конкретном примере. Построенная в статье зависимость пути торможения от начальной скорости вагона позволяет судить об ее возрастающем квадратичном характере. Приведен анализ формуль пути торможения вагона с позиции классической механики, который доказывает корректность и применимость построенных математических моделей применительно к зоне торможения вагона на всех участках тормозных позиций.

Цель - построить математическую модель движения вагона на участках тормозных позищий сортировочных горок. 
Метод или методология проведения работы: математическое описание движения вагона на участках тормозных позиций сортировочной горки осуществлено с использованием теоремь об изменении кинетической энергии для несвободной материальной точки в конечной форме.

Результаты: получена формула пути торможения вагона на участках тормозных позиций сортировочных горок.

Область применения результатов: результаты исследований по определению пути торможения вагона в зоне затормаживания вагона могут быть использованы при проектировании профиля пути сортировочной горки.

Ключевые слова: железная дорога; железнодорожная станция; сортировочная горка; вагон; тормозная позиция; путь торможения.

\section{ABOUT THE APPROACH TO THE DETERMINATION OF CERTAIN KINEMATIC PARAMETERS OF THE CAR'S MOVEMENT ON THE HUMP BRAKING POSITIONS OF MARSHALLING YARDS}

\section{Turanov Kh.T., Gordienko A.A., Saidivaliev Sh.U.}

In the design of marshalling hump, the choice of optimal parameters of great importance to have accurate values of the kinematic parameters of the movement of the cars, uncoupling at all parts. The analysis of the existing method of calculation of marshalling humps shows that the formulas used contain a significant number of inaccuracies and does not contain some expressions for determining important kinematic values. The article presents a mathematical description of the movement of the car on the sections of the hump braking positions of the marshalling hump using the theorem on the change of kinetic energy for a non-free material point in the final form. This approach makes it possible to solve the previously intractable problem of deriving the formula of the braking path of the car on the sections of the hump brak- 
ing positions of the marshalling humps. The resulting mathematical model is tested on a specific example. The dependence of the braking path on the initial speed of the car built in the article allows to judge its increasing quadratic character. The analysis of the formula of the braking path of the car from the position of classical mechanics, which proves the correctness and applicability of the constructed mathematical models in relation to the braking section of the car in all parts of the hump braking positions.

Purpose. To build a mathematical model of the movement of the car on the sections of the hump braking positions of the marshalling stations.

Methodology. The mathematical description of the movement of the car on sections of hump braking positions of the marshalling stations is carried out using of the theorem of changing of kinetic energy for a non-free material point in a final form.

Results: for the first time, the formula of the braking path of the car on the sections of the hump braking position of the marshalling stations was obtained.

Practical implications the results of studies to determine the braking path of the car in the area of braking of the car can be used in the design of the profile of the path of the marshalling hump.

Keywords: railway; railway station; marshalling hump; wagon; hump braking position; braking path.

Настоящая статья является продолжением серии публикаций по проблеме расчёта и проектирования профиля пути сортировочных горок [1-16].

Интересно заметить, что в $[3,15]$ для определения энергетической высоты сортировочной горки $\boldsymbol{h}_{\mathrm{h}}$ используется одна и та же методика. В них принято допущение о том, что в любой точке наклонной плоскости энергия скатывающегося тела массой $\boldsymbol{M}$ равна сумме потенциальной $\boldsymbol{E}_{\mathrm{p}}$ и кинетической энергии $\boldsymbol{E}_{\mathbf{c}}$. Предполагается, что эта энергия расходуется на работу сил сопротивления движению $\boldsymbol{A}_{\mathbf{r}}$, т.е. $\boldsymbol{E}_{\mathbf{c}}+\boldsymbol{E}_{\mathbf{p}}=\boldsymbol{A}_{\mathbf{r}}$ (см. стр. 8 в [3] и формулу (6) в [15]). 
Отсюда определяют энергетическую высоту сортировочной горки $\boldsymbol{h}_{\mathbf{h}}$. Однако, такой подход противоречит закону сохранения энергии $[6,7]$. До настоящего времени проектирование энергетической высоты сортировочной горки $\boldsymbol{h}_{\mathrm{h}}$ выполняется с использованием понятия «мощность тормозных позиций $\boldsymbol{h}_{\mathrm{br}}$ или тормозных средств $\boldsymbol{h}_{\text {brt }}{ }^{\prime}[1,2,3,5,15]$. Мощность тормозных позиций $\boldsymbol{h}_{\mathrm{br}}$ выбирают согласно [5]. Энергетическая высота $\boldsymbol{h}_{\mathrm{h}}$ сортировочной горки в пределах расчётной длины (от вершины горки (НР) до расчётной точки (СТ)) определяют как сумму трех профильных высот расчетных участков (см. рисунок 5.1 в [5]): головного участка (между вершиной горки и началом первой тормозной позиций (1BP)) $\boldsymbol{h}_{\mathrm{h} 1}$, среднего участка (между началом 1 тормозной позицией $1 \mathrm{BP}$ и началом пучковой тормозной позиции (2ВР)) $\boldsymbol{h}_{\mathrm{h} 2}$ и нижнего участка (между началом пучковой тормозной позиции $2 \mathrm{BP}$ и расчетной точкой СТ) $\boldsymbol{h}_{\mathrm{h} 3}$. При этом значения $\boldsymbol{h}_{\mathrm{h} 1}, \boldsymbol{h}_{\mathrm{h} 2}$ и $\boldsymbol{h}_{\mathrm{h} 3}$ вычисляют по эмпирической формуле (см. формула (5.1) в [5]):

$$
h_{1}^{\max }=\frac{v_{b x}^{2}-v_{0}^{2}}{2 \mathrm{~g}_{0 \mathrm{x}}}+h_{\text {ock1 }}+h_{c \kappa 1} .
$$

Эта формула состоит из трех слагаемых. Первое слагаемое в формуле (5.1) [5] получено при отсутствии силы связи между колесами вагона и рельсовыми нитями (т.е. для идеальной связи), когда полная кинетическая энергия вагона при его перемещении из СТ на некоторое расстояние равно работе составляющей силы тяжести на направление перемещения вагона $(\boldsymbol{G} \sin \psi)$ по рельсовым нитям [5]. Вторые и третьи слагаемые формулы (5.1) в [5] представляют собой, предположительно, потери удельной энергии при преодолении основного удельного сопротивления движению и сопротивления стрелок и кривых в пределах соответствующих участков $\boldsymbol{h}_{\mathrm{h} 1}, \boldsymbol{h}_{\mathrm{h} 2}$ и $\boldsymbol{h}_{\mathrm{h} 3}$ [5]. Иначе, формулы для определения $\boldsymbol{h}_{\mathrm{h} 1}$, $\boldsymbol{h}_{\mathrm{h} 2}$ и $\boldsymbol{h}_{\mathrm{h} 3}$ применимы для неидеальных связей. Такая же методика расчёта прослеживается в [3, 15].

Согласно первому слагаемому формулы (5.1) [5], колеса вагона скользят по рельсовым нитям. Это не соответствует реально- 
му движению колёс колесных пар вагона по рельсовым нитям, поскольку последние невозможно представить в виде идеальных связей. Согласно вторым и третьим слагаемым формулы (5.1) [5] колеса вагона катятся без скольжения по рельсовым нитям. В действительности профиль сортировочной горки (наклонная плоскость) является неидеальной (негладкой и/или с трением) поверхностью и вагон с грузом движется по профилю горки с начальной скоростью $\boldsymbol{v}_{\mathbf{0}}$, равной нулю (т.е. $\boldsymbol{v}_{\mathbf{0}} \neq 0$ ).

Несмотря на это, все результаты исследований по проектированию сортировочной горки в $[1,2,4,5,15,16]$ получены:

во-первых, с учётом инерции вращающихся частей (колёсных пар), включая участки тормозных позиций, на которых происходит поступательное движение колёсных пар. Здесь недопустимым является учет инерции вращающихся частей на участках тормозных позиций, так как наблюдается чистое скольжение колёс относительно рельсовых нитей и тормозных шин вагонного замедлителя;

во-вторых, для неидеальных поверхностей, с учётом профиля сортировочных горок;

в-третьих, при определении скорости движения вагона на всех участках горки, включая тормозные позиции, по формуле $v=\sqrt{2 \mathrm{~g}^{\prime} h}$ (где $\mathrm{g}^{\prime}-$ ускорение свободно падающего тела с учётом инерции вращающихся частей) (см. стр. 186 в [1]), применительно для идеальной поверхности, что недопустимо.

Как видно, ошибочность определения энергетической высоты сортировочной горки $\boldsymbol{h}_{\mathrm{h}}$ состоит в использовании несовместимого по физическому смыслу понятия идеальной связи к решению задач сортировочных горок, на которой фактически связи являются неидеальными.

До сих пор авторы статьи [11-14] считали решение транспортной задачи по определению времени движения и пути торможения вагона на участках тормозных позиций сортировочных горок трудноразрешимой задачей. В существующей методике горочных конструктивных и технологических расчётов в [4] такую задачу рекомендуют решить с использованием понятия «мощности тор- 
мозных позиций». Здесь ошибка состоит в использовании формулы свободного падения тела $v=\sqrt{2 \mathrm{~g}^{\prime} h}$, применимой только для идеальной связи [5].

Таким образом, выявлено, что проблема математического моделирования участков тормозных позиций сортировочных горок до сих пор не решена.

\section{Принятые допущения}

Предположим, что любые точки вагона, включая его центр масс $\boldsymbol{C}_{\text {в }}$ и центр масс $\boldsymbol{C}$ колёсных пар (здесь рисунок не приводятся), в зонах торможения участков тормозных позиций будут двигаться с одинаковыми скоростями, равными скорости входа вагона $\boldsymbol{v}_{\text {вх.г }}=$ $v_{0}=v_{\text {нт }}$ В эту зону, т.е. $v_{C_{\mathrm{B}}}=\boldsymbol{v}_{C}=\boldsymbol{v}_{\mathrm{B} \times \mathrm{T}}=\boldsymbol{v}_{\mathbf{0}}=\boldsymbol{v}_{\mathrm{uт}}=$ const $>0$. ПоэТОМУ движение вагона с грузом $\boldsymbol{G}$ в зонах затормаживания можно рассматривать как движение системы материальных точек, предполагая всю его массу $\boldsymbol{M}_{\text {в }}$ сосредоточенной в его центре масс $\boldsymbol{C}_{\mathrm{B}}$. При этом неподвижную систему отсчёта расположим в то положение, в котором находилась точка $\boldsymbol{C}_{\text {в }}$ вагона в начале его затормаживания. Поэтому за начало отсчёта времени, т.е. за начальный момент $\boldsymbol{t}=$ 0 , возьмем момент начала затормаживания вагона, движущегося по уклону сортировочной горки с постоянной скоростью $\boldsymbol{v}_{\text {нт }}=\boldsymbol{v}_{\text {вх.т }}$ $=\boldsymbol{v}_{\mathrm{k}}=$ const $>0$.

\section{Математическое описание решение задачи}

Запишем теорему об изменении кинетической энергии для несвободной материальной точки на перемещении $\boldsymbol{A B}$, между которыми возможно движение вагона, с учётом начальной скорости $\boldsymbol{v}_{\text {н.т }}$ в конечной форме $[5,6]$ применительно к решению рассматриваемой задачи в виде:

$$
\frac{G}{2 \mathrm{~g}}\left(v_{k \cdot \mathrm{T}}^{2}-v_{\mathrm{H. \textrm {T }}}^{2}\right)=A_{F x},
$$

с учётом того, что в ней

$$
A_{F x}=A_{G x}+A_{F \mathrm{Tp}},
$$


где

$\boldsymbol{A}_{\boldsymbol{G}}-$ работа проекции силы тяжести $\boldsymbol{G}_{\boldsymbol{x}}$ по оси $O x$, проведенной параллельно рельсовым нитям, на перемещении $\boldsymbol{x}_{\text {вв }}$ между точками $\boldsymbol{A}$ и $\boldsymbol{B}$, произведённой силой $\boldsymbol{G}_{\boldsymbol{x}}$ :

$$
A_{G x}=G_{x} x_{C_{\mathrm{B}}}=G \sin \psi x_{C_{\mathrm{B}}} ;
$$

$\boldsymbol{A}_{\boldsymbol{F} \text { тр }}-$ работа силы трения $\boldsymbol{F}_{\text {тр }}$ (в общем случае, может быть и силы сопротивлений всякого рода $\boldsymbol{F}_{\mathrm{c}}$ ) на перемещении $\boldsymbol{x}_{\boldsymbol{C}_{\mathrm{B}}}$ между точками $\boldsymbol{A}$ и $\boldsymbol{B}$ :

$$
A_{F \mathrm{Tp}}=-F_{\text {тр }} x_{C_{\mathrm{B}}}=-f_{\mathrm{T}} G \cos \psi x_{C_{\mathrm{B}}} .
$$

Подставляя последние две формулы в (3) с учётом (2), после упрощений, можно получить формулу для определения скорости движения вагона в зоне торможения на участках тормозных позиций:

$$
v_{k . \mathrm{T} i}^{2}-v_{\mathrm{H} . \mathrm{T} i}^{2}=2 \mathrm{~g}\left(\sin \psi_{\mathrm{T} i}-f_{\mathrm{T}} \cos \psi_{\mathrm{T} i}\right) x_{C_{\mathrm{B} i} i}
$$

или при $\boldsymbol{x}_{\mathrm{Cв} i}=\boldsymbol{l}_{\text {ті }}$

$$
v_{k . \mathrm{Ti}}^{2}=v_{\mathrm{H.Ti}}^{2}+2 \mathrm{~g}\left(\sin \psi_{\mathrm{\tau} i}-f_{\mathrm{T}} \cos \psi_{\mathrm{T} i}\right) l_{\mathrm{T} i} .
$$

где $\boldsymbol{i}$ - номера участков профиля пути $(\boldsymbol{i}=1, \ldots 9)$.

Отсюда, при $\boldsymbol{v}_{k . \mathrm{Ti}}=0$ и $\boldsymbol{x}_{\text {Свi }}=\boldsymbol{l}_{\text {ті }}$,

$$
0=v_{\text {н.тi }}^{2}+2 \mathrm{~g}\left(\sin \psi_{\mathrm{т} i}-f_{\mathrm{T}} \cos \psi_{\mathrm{т} i}\right) l_{\mathrm{T} i} .
$$

Из последнего равенства окончательно получим путь затормаживания вагона $\boldsymbol{x}_{\boldsymbol{C} \boldsymbol{i} i}=\boldsymbol{l}_{\text {ті }}$ :

$$
l_{\mathrm{r} i}=\frac{v_{\mathrm{H. \textrm {T }}}^{2}}{2 \mathrm{~g}\left(f_{\mathrm{T}} \cos \psi_{\mathrm{T} i}-\sin \psi_{\mathrm{T} i}\right)} .
$$

Если иметь в виду, что для малых углов (менее $5^{\circ}$ ), соответствующих профилю на всей протяженности пути сортировочной горки: $\sin \psi_{i} \approx \boldsymbol{\psi}_{\boldsymbol{i}}=\boldsymbol{i}, \boldsymbol{\operatorname { c o s }} \boldsymbol{\psi}_{\boldsymbol{i}} \approx 1$, то формулы (6) и (7) примут вид:

$$
\begin{gathered}
v_{k . \mathrm{T} i}^{2}=v_{\mathrm{н} . \mathrm{T} i}^{2}+2 \mathrm{~g}\left(i_{i}-f_{\mathrm{T}}\right) l_{\mathrm{T} i} ; \\
l_{\mathrm{T} i}=\frac{v_{\mathrm{H. \textrm {T }} i}^{2}}{2 \mathrm{~g}\left(f_{\mathrm{T}}-i_{i}\right)} .
\end{gathered}
$$

Как видно, величина тормозного пути $\boldsymbol{l}_{\text {затi }}$ (и/или $\boldsymbol{l}_{\text {ті }}$ ) прямо пропорциональна квадрату начальной скорости $\boldsymbol{v}_{\text {нті }}$ и обратно пропор- 
ционально коэффициенту трения скольжения $\boldsymbol{f}_{\mathbf{\tau}}$. и уклона профиля пути $\boldsymbol{i}_{\boldsymbol{i}}$.

Таким образом, применение теоремы об изменении кинетической энергии материальной точки в конечном виде $[5,6]$ в зонах торможения вагона на участках тормозных позиций по формуле (7) и/ или (9) позволили определить путь торможения вагона $\boldsymbol{l}_{\text {затi }}\left(\right.$ и/или $\left.\boldsymbol{l}_{\text {тi }}\right)$.

При этом, для вычисления $\boldsymbol{l}_{\text {ті }}$ рассматриваются следующие варианты:

а) непосредственный вход на участок тормозной позиции первой колёсной пары $\boldsymbol{l}_{\text {вх } \boldsymbol{i}}$ и/или колёсных пар передней тележки $\boldsymbol{l}_{\text {пт }}$;

в) вход вагона на участок на длину базы вагона $\boldsymbol{l}_{\text {кб̃ }}$, которые необходимы для задания начальной скорости $v_{\text {ні:т }}$ и/или скорости входа вагона $\boldsymbol{v}_{\text {вх } i}$ (имея в виду, что $\boldsymbol{v}_{\text {ні.т }}=\boldsymbol{v}_{\text {вхі.т }}$ ) в зону затормаживания.

Интересно заметить, что, если, известно значение ускорения движения при равнозамедленном движении вагона $\left|\boldsymbol{a}_{\text {тi }}\right|=-\boldsymbol{a}_{\text {ті }}$ по силовым соотношениям, то по формуле скорости элементарной физики

$$
v_{\mathrm{T} i}=v_{\mathrm{H} i}+\left|a_{\mathrm{T} i}\right| t_{\mathrm{T} i}
$$

можно найти время затормаживания $\boldsymbol{t}_{\text {затi }}=\boldsymbol{t}_{l \mathbf{}}$ до момента остановки вагона $\boldsymbol{t}_{\text {затi }}=\boldsymbol{t}_{l \boldsymbol{i} i}<\boldsymbol{t}$, где $\boldsymbol{t}-$ текущее время в секундах:

$$
t_{\mathrm{T} i}=\frac{v_{\mathrm{H}}-v_{\mathrm{T} i}}{\left|a_{\mathrm{Ti} i}\right|} .
$$

Для сравнения по значению времени затормаживания $\boldsymbol{t}_{\text {затi }}=\boldsymbol{t}_{l \boldsymbol{i} i}$ можно найти путь торможения вагона $\boldsymbol{l}_{\text {т }}$ с использованием формулы пути элементарной физики на исследуемом участке тормозных позиций.

Таким образом, формулы скорости элементарной физики при заданном значении ускорения при равнозамедленном движении вагона $\left|\boldsymbol{a}_{\text {тi }}\right|$, полученным по силовым соотношениям, позволили найти время затормаживания вагона $\boldsymbol{t}_{\text {затi }}=\boldsymbol{t}_{l \mathbf{i} i}$ до момента остановки вагона, т.е. при $\boldsymbol{t}_{\text {затi }}=\boldsymbol{t}_{l \boldsymbol{i} i}<\boldsymbol{t}$, где $\boldsymbol{t}$ - текущее время в секундах.

Особо оговоримся, что время затормаживания вагона $\boldsymbol{t}_{\text {ті }}$, вычисленное по формуле (11), имеет отрицательный знак, который означает замедленное движение на участках тормозных позиций и то, что $\boldsymbol{t}_{\text {тi }}<\boldsymbol{t}(\boldsymbol{t}-$ текущее время). 


\section{Исходные данные и результаты}

Для примера рассмотрим участок второй тормозной позиций (2ТП) сортировочной горки. Исходные данные: для малых углов $\sin \boldsymbol{\psi}_{2 \mathrm{~T}} \approx \boldsymbol{\psi}_{2 \mathrm{~T}}=0,010-$ уклон профиля пути, рад., или $\boldsymbol{i}_{2 \mathrm{~T}}=10 \% ; \boldsymbol{G}=$ 650 сила тяжести груза на вагоне, кH; $\boldsymbol{G}_{\boldsymbol{1}}=794$ - сила тяжести вагона с грузом совместно с невращающимися частями (кузов вагона, тележка и колёсные пары), кH; $\boldsymbol{F}_{x 2}=11,13$ - проекции силы воздействующие на участке торможения с учётом силы попутного ветра малой величины $\left(\boldsymbol{F}_{\text {в } \boldsymbol{x}}=3,2\right.$ кН $), \mathbf{\kappa H} ;\left|\boldsymbol{F}_{\mathbf{c} 2}\right|=-\boldsymbol{F}_{\mathbf{c} 2} \approx-222,84-$ модуль силы сопротивления всякого рода (учёт силы нажатия тормозных колодок вагонных замедлителей на обода колёс вагона при скорости входа вагона в зону затормаживания $\left.\boldsymbol{v}_{\text {вх.т }} \leq 6,5 \mathrm{~m} / \mathrm{c}\right)$, кН; $\boldsymbol{M}_{\text {пр0 }}=8,869 \cdot 10^{4}-$ приведённая масса вагона с грузом совместно с невращающимися частями, кг; $\boldsymbol{v}_{\text {нт2 }}=\boldsymbol{v}_{\text {вх.т2 }}=3,879 \approx 3,88-$ начальная скорость и/или скорость входа вагона в зону затормаживания участка 2ТП, м/с.

Результаты расчёта [7]. Путь торможения вагона на участке второй тормозной позиции сортировочной горки $\boldsymbol{l}_{\mathrm{r2}}$, вычисленный согласно формуле (10), оказался равным: $\boldsymbol{l}_{\mathrm{r} 2}=3,195$ м. При этом практически происходит полная остановка вагона, т.е. $\boldsymbol{v}_{\text {т2 }}=0$.

Расчётное значение ускорения при равнозамедленном движении вагона $\left|\boldsymbol{a}_{\text {тi }}\right|$, полученное согласно силовым соотношениям $\boldsymbol{F}_{\boldsymbol{x} 2}$ и $\left|\boldsymbol{F}_{\mathbf{c} 2}\right|$, оказалось равным $\left|\boldsymbol{a}_{\text {ті }}\right|=2,387 \mathrm{~m} / \mathrm{c}^{2}$.

При расчёте скорости движения вагона $v_{\mathbf{T} 2}$ по формуле (10) при $\boldsymbol{l}_{\mathrm{\tau} 2}=3,195$ м и начальной скорости $\boldsymbol{v}_{\mathrm{H} 2}=3,879$ м/с получился комплексный результат: $\boldsymbol{v}_{\text {т2 }}=\mathbf{I}\left(\boldsymbol{v}_{\mathrm{T2}}\right)=0,456 \boldsymbol{i} \mathrm{M} / \mathrm{c}-$ мнимая часть числа $\boldsymbol{v}_{\mathbf{r 2}}$, где $\boldsymbol{i}$ - мнимая единица. Это означает, что действительная часть $\mathbf{R}\left(\boldsymbol{v}_{\text {т2 }}\right)=0$. В свою очередь, это подтверждает корректность вывода аналитической формулы (7) и/или (9).

Время торможения вагона $\boldsymbol{t}_{\mathbf{2} 2}$ по формуле (11) скорости элементарной физики равно: $\boldsymbol{t}_{\mathrm{\tau} 2}=1,625$ с до момента остановки вагона, когда $\boldsymbol{v}_{\mathrm{т2}}=0$, т.е. при $\boldsymbol{t}_{\mathrm{т2}}<\boldsymbol{t}$, где $\boldsymbol{t}-$ текущее время в секундах. Например, при $\boldsymbol{t}=1 \mathrm{c}: \boldsymbol{v}_{\mathrm{r} 2}=1,492 \mathrm{M} / \mathrm{c}$; при $\boldsymbol{t}=1,2 \mathrm{c}: \boldsymbol{v}_{\mathrm{r} 2}=1,05 \mathrm{м} / \mathrm{c} ;$ при $\boldsymbol{t}=1,4$ с: $\boldsymbol{v}_{\mathrm{T} 2}=0,535 \mathrm{м} / \mathrm{c}$; при $\boldsymbol{t}=1,5 \mathrm{c}: \boldsymbol{v}_{\mathrm{r} 2}=0,299 \mathrm{м} / \mathrm{c} ;$ при $\boldsymbol{t}=1,6 \mathrm{c}$ : $v_{\mathrm{T} 2}=0,06 \mathrm{M} / \mathrm{c}$. 
Как видно, формула (10) может быть использована для определения скорости скольжения вагона до момента его остановки в зоне торможения на участках тормозных позиций сортировочной горки.

Значение пути торможения вагона $\boldsymbol{l}_{\text {ті, }}$ определенная с использованием формулы пути элементарной физики на исследуемом участке тормозных позиций при времени затормаживания $\boldsymbol{t}_{\mathrm{т} 2}=1,625 \mathrm{c}$, оказалось равным $\boldsymbol{l}_{\text {т02 }}=3,152$ м. Относительная ошибка расчета сравнительно с формулой (6) составила 1,37 \%, что ничтожно мало.

Графическая зависимость $l_{\text {т2 }}=f\left(v_{\text {нт2 }}\right)=f\left(v_{02 \mathrm{~T}}\right)$ и/или $l_{\mathrm{b} 2}=f\left(v_{\text {i2b }}\right)$, построенная, согласно формуле (7) и формуле элементарной физики (10), при вариации $\boldsymbol{v}_{\mathbf{0 2 \mathrm { T }}}=\boldsymbol{v}_{\mathrm{i} 2 \mathrm{~b}}$ от 0 до 5 с шагом $\Delta \boldsymbol{v}_{\mathrm{i} 2 \mathrm{~b}}=0,25 \mathrm{M} / \mathrm{c}$, представлена на рисунке 1 .

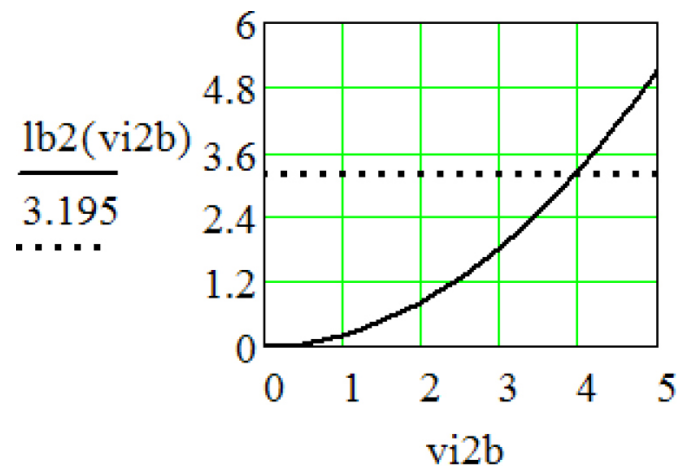

Рис. 1. Графическая зависимость $l_{\mathrm{b} 2}=f\left(v_{02 \mathrm{~T}}\right)$

Из рисунка 1 ясно, что характер зависимости пути торможения от начальной скорости вагона имеет вид возрастающей квадратичной зависимости.

Как видно, при $\boldsymbol{v}_{\text {н2 }}=\boldsymbol{v}_{\text {вх.2т }}=0$ путь торможения $\boldsymbol{l}_{\text {зат2 }}=\boldsymbol{l}_{\mathrm{\tau} 2}=0$. Это подтверждает рассуждение о важности входа вагона в зоны торможения тормозных позиций с начальной скоростью $\boldsymbol{v}_{\text {н2 }}=\boldsymbol{v}_{\text {вх.2т }}>0$, в противном случае происходит полная остановка вагона до включения вагонного замедлителя. При выполнении условия $\boldsymbol{v}_{\text {н2 }}=\boldsymbol{v}_{\text {вх.2т }}>0$ кинетическая энергия $\boldsymbol{E}_{\boldsymbol{k}}=\boldsymbol{E}_{\mathbf{0}}$ вагона с массой $\boldsymbol{M}$ и начальной скоростью $\boldsymbol{v}_{\text {н2 }}$ будет полностью израсходована на преодоления работы 
$\boldsymbol{A}_{\boldsymbol{r}}$ сил сопротивления $\boldsymbol{F}_{\boldsymbol{c}}$, появляющегося при включении вагонного замедлителя. В свою очередь, работа $\boldsymbol{A}_{\mathbf{c}}$ будет рассеиваться в окружающую среду в виде тепла. При полной остановке вагона, т.е. $\boldsymbol{v}_{\text {к.2т }}=0$, будет соблюдено условие: $\boldsymbol{E}_{\mathbf{0}}+\left(-\boldsymbol{A}_{\mathrm{c}}\right)=0$.

Таким образом, результаты расчётов пути торможения $\boldsymbol{l}_{\text {зат }}$ вагона с использованием выражения (7) и формулы пути элементарной физики, позволили отметить, что при одном и том же значении начальной скорости $\boldsymbol{v}_{\text {н2 }}=\boldsymbol{v}_{\text {вх.2т }}$, они дают одинаковые данные.

В свою очередь, это подтверждает неоспоримость, корректность и применимость построенных математических моделей применительно к зоне торможения вагона на всех участках тормозных позиций.

\section{Заключение}

1. С использованием теоремы об изменении кинетической энергии для несвободной материальной точки в конечной форме впервые решена проблема математического моделирования кинематических параметров движения вагона в зонах торможения на участках тормозных позиций сортировочных горок железных дорог.

2. Результаты расчётов пути торможения вагона на тормозных позициях сортировочных горок с использованием предложенных авторами статьи формул и формулы пути элементарной физики позволили отметить, что при одном и том же значений начальной скорости, они дают одинаковые результаты. Это подтверждает неоспоримость, корректность и применимость построенных математических моделей применительно к зоне торможения вагона на всех участках тормозных позиций.

\section{Список литературы}

1. Образцов В.Н.. Станции и узлы. ч. II. М: Трансжелдориздат, 1938. $492 \mathrm{c}$.

2. Образцов В.Н., Никитин В.Д., Шаульский Ф.И., Бузанов С.П. Станции и узлы. М: Трансжелдориздат, 1949. 540 c. 
3. Акулиничев В.М., Колодий Л.П. Расчет и проектирование горок большой и средней мощности. М: Транспорт, 1981. 68 с.

4. Апатцев В.И., Ефименко Ю.И. Железнодорожные станции и узлы: учебник. М: ФГБОУ «Учебно-методический центр по образованию на железнодорожном транспорте», 2014. 332 с.

5. Правила и нормы проектирования сортировочных устройств на железных дорогах колеи 1520 мм. М: ТЕХИНФОРМ, 2003. 168 с.

6. Тимошенко С.П., Юнг Д. Инженерная механика. М: Машгиз, 1960. $508 \mathrm{c}$.

7. Тарг С.М. Краткий курс теоретической механики: учебник для втузов. М: Высшая школа, 1998. 416 с.

8. Шмаль С.Н. Попытка заново рассчитать брахистохрону // Мир транспорта. 2011. №3. С. 34-38.

9. Кобзев В.А., Шмаль С.Н. Особенности расчёта спускной части сортировочных горок методом покоординатного спуска // Наука и техника транспорта. 2014. №1. С. 17-20.

10. Долженко А.М. Оптимальное управление роспуском поездов на сортировочных горках малой мощности // Вестник транспорта Поволжья. 2014 №1 (43). С. 56-60.

11. Туранов Х.Т., Гордиенко А.А., Мягкова А.В. Уточненные результаты вычислений времени движения и скорости вагона на втором скоростном участке сортировочной горки при воздействии попутного ветра // Известия Петербургского университета путей сообщения. 2016, № 1 (46). С. 40-49.

12. Turanov Kh. Analytical investigation of wagon speed and traversed distance during wagon hump rolling under the impact of gravity forces and head wind // Global Journal of Researches in Engineering: A. Mechanical and Mechanics Engineering. 2014. Volume 14 Issue 1 Version 1.0 Year 2014. New York, pp. 1-9.

13. Turanov Kh., Gordienko A., Myagkova A. Analytical Description of Wagon Motion on the Second Speed Section of the Marshalling Hump with Switch Zone under the Impact of Fair Wind // Journal of Multidisciplinary Engineering Scince and Technology (JMEST). 2015. Vol. 2 Issue 11, November-2015, pp. 3255-3262. 
14. Turanov Kh., Gordienko A., Plakhotich I. Simplified Analytical Description of Wagon Movement with Braking Action on the Marshalling Hump Section of the First Braking Position under the Impact of Fair Wind // Science and Technology. 2015. Vol, 5 No. 4, December 2015, pp. 57-62. doi: 10.5923/j.scit.2015 04.01

15. Prokop J., Myojin Sh. Desing of Hump Profile in Railroad Classification Yard // Memoirs of the Faculty of Engineering. Okayama University. 1993. Vol. 27. No. 2, pp. 41-58.

16. Prokop J., Myojin Sh. Simulation of Hump Perfomancre in Railroad Classification Yard // Memoirs of the Faculty of Engineering. Okayama University. 1993. Vol. 27. No. 2, pp. 59-71.

\section{References}

1. Obraztsov V.N. Stantsii $i$ uzly [Stations and nodes]. Part II. M: Transzheldorizdat, 1938. $492 \mathrm{p}$.

2. Obraztsov V.N., Nikitin V.D., Shaul'skii F.I., Buzanov S.P. Stantsii $i$ uzly [Stations and nodes]. M: Transzheldorizdat, 1949. 540 p.

3. Akulinichev V.M., Kolodii L.P. Raschet i proektirovanie gorok bol'shoi $i$ srednei moshchnosti [Calculation and design of high and medium capacity slides]. M: Transport, 1981. $68 \mathrm{p}$.

4. Apattsev V.I., Efimenko Iu.I. Zheleznodorozhnye stantsii i uzly [Railway stations and nodes]. M: FGBOU «Uchebno-metodicheskii tsentr po obrazovaniiu na zheleznodorozhnom transporte», 2014. $332 \mathrm{p}$.

5. Pravila i normy proektirovaniia sortirovochnykh ustroistv na zheleznykh dorogakh kolei $1520 \mathrm{~mm}$ [Rules and regulations for the design of sorting devices on railways with a gauge of $1,520 \mathrm{~mm}]$. M: TEKHINFORM, 2003. $168 \mathrm{p}$.

6. Timoshenko S.P., Iung D. Inzhenernaia mekhanika [Engineering Mechanics]. M: Mashgiz, 1960. 508 p.

7. Targ S.M. Kratkii kurs teoreticheskoi mekhaniki [A short course in theoretical mechanics: a textbook for technical colleges]. M: Vysshaia shkola, 1998. 416 p.

8. Shmal' S.N. Mir transporta. 2011. No 3, pp. 34-38.

9. Kobzev V.A., Shmal' S.N. Nauka i tekhnika transporta. 2014. No 1, pp. 17-20. 
10. Dolzhenko A.M. Vestnik transporta Povolzh'ia. 2014. No 1 (43), pp. 56-60.

11. Turanov Kh.T., Gordienko A.A., Miagkova A.V. Izvestiia Peterburgskogo universiteta putei soobshcheniia. 2016, no 1 (46), pp. 40-49.

12. Turanov Kh. Analytical investigation of wagon speed and traversed distance during wagon hump rolling under the impact of gravity forces and head wind. Global Journal of Researches in Engineering: A. Mechanical and Mechanics Engineering. 2014. Volume 14 Issue 1 Version 1.0 Year 2014. New York, pp. 1-9.

13. Turanov Kh., Gordienko A., Myagkova A. Analytical Description of Wagon Motion on the Second Speed Section of the Marshalling Hump with Switch Zone under the Impact of Fair Wind. Journal of Multidisciplinary Engineering Scince and Technology (JMEST). 2015. Vol. 2 Issue 11, November - 2015, pp. 3255-3262.

14. Turanov Kh., Gordienko A., Plakhotich I. Simplified Analytical Description of Wagon Movement with Braking Action on the Marshalling Hump Section of the First Braking Position under the Impact of Fair Wind. Science and Technology. 2015. Vol, 5 No. 4, December 2015, pp. 57-62. doi: 10.5923/j.scit.2015 04.01

15. Prokop J., Myojin Sh. Desing of Hump Profile in Railroad Classification Yard. Memoirs of the Faculty of Engineering. Okayama University. 1993. Vol. 27. No. 2, pp. 41-58.

16. Prokop J., Myojin Sh. Simulation of Hump Perfomancre in Railroad Classification Yard. Memoirs of the Faculty of Engineering. Okayama University. 1993. Vol. 27. No. 2, pp. 59-71.

\section{ДАННЫЕ ОБ АВТОРАХ}

Туранов Хабибулла Туранович, профессор кафедры «Станции, узлы и грузовая работа», доктор технических наук Уральский государственный университет путей сообщеения

ул. Колмогорова, 66, г. Екатеринбург, 620034, Российская Федерачия

khturanov@yandex.ru 
Гордиенко Андрей Александрович, доцент кафедры «Станции, узлы и грузовая работа», кандидат технических наук Уральский государственный университет путей сообщения ул. Колмогорова, 66, г. Екатеринбург, 620034, Российская Федерация gordiii89@yandex.ru

Саидивалиев Шухрат Умарходжаевич, ассистент кафедры «Транспортная логистика и сервис» Ташкентский институт инженеров железнодорожного транспорта

Temirylchilar 1-tor kycha, 1, ТашИИТ, г. Ташкент, 100167, Узбекистан

khturanov@yandex.ru

\section{DATA ABOUT THE AUTHORS}

Turanov Khabibulla Turanovich, Professor «Station, Junctions and Freight Work», Doctor of Technical Sciences

Ural State University of Railway Transport

66, Kolmogorova St., Ekaterinburg, 620034, Russian Federation khturanov@yandex.ru

SPIN-code: 8922-6207

Gordienko Andrey Aleksandrovich, Assistant Professor «Station, Junctions and Freight Work», Candidate of Technical Sciences 66, Kolmogorova St., Ekaterinburg, 620034, Russian Federation Gordiii89@yandex.ru

SPIN-code: 8073-2014

Saidivaliev Shukhrat Umarkhodjaevich, Assistant "Transport Logistics and Service"

Tashkent Institute of Railway Engineers

Temirylchilar 1-tor kycha, 1, TIRE, Tashkent, 100167, Uzbekistan khturanov@yandex.ru 